\title{
Evaluation of Visible-Near Infrared Reflectance Spectra of Avocado Leaves as a Non-destructive Sensing Tool for Detection of Laurel Wilt
}

\author{
Sindhuja Sankaran and Reza Ehsani, Citrus Research and Education Center, University of Florida, Lake Alfred 33850; and Sharon \\ A. Inch and Randy C. Ploetz, Tropical Research and Education Center, University of Florida, Homestead 33031
}

\begin{abstract}
Sankaran, S., Ehsani, R., Inch, S. A., and Ploetz, R. C. 2012. Evaluation of visible-near infrared reflectance spectra of avocado leaves as a nondestructive sensing tool for detection of laurel wilt. Plant Dis. 96:1683-1689.

Laurel wilt, caused by the fungus Raffaelea lauricola, affects the growth, development, and productivity of avocado, Persea americana. This study evaluated the potential of visible-near infrared spectroscopy for non-destructive sensing of this disease. The symptoms of laurel wilt are visually similar to those caused by freeze damage (leaf necrosis). In this work, we performed classification studies with visible-near infrared spectra of asymptomatic and symptomatic leaves from infected plants, as well as leaves from freeze-damaged and healthy plants, both of which were non-infected. The principal component scores computed from principal component analysis were used as input features in four classifiers: linear discriminant analysis, quadratic

discriminant analysis (QDA), Naïve-Bayes classifier, and bagged decision trees (BDT). Among the classifiers, QDA and BDT resulted in classification accuracies of higher than $94 \%$ when classifying asymptomatic leaves from infected plants. All of the classifiers were able to discriminate symptomatic-infected leaves from freeze-damaged leaves. However, the false negatives mainly resulted from asymptomatic-infected leaves being classified as healthy. Analyses of average vegetation indices of freeze-damaged, healthy (non-infected), asymptomaticinfected, and symptomatic-infected leaves indicated that the normalized difference vegetation index and the simple ratio index were statistically different.
\end{abstract}

Laurel wilt is a serious disease of avocado (Persea americana) and other tree species in the family Lauraceae, such as redbay (Persea borbonia). The causal fungus, Raffaelea lauricola, is vectored by an invasive ambrosia beetle, Xyleborus glabratus, which was first reported in the United States in 2002 in Port Wentworth, GA (4). Laurel wilt was observed shortly afterward on redbay trees in the vicinity. In Florida, laurel wilt was first observed in Duval County in 2005 on redbay, and on avocado in 2006 (18). Since then, the disease has spread throughout the state, reaching the Florida panhandle in 2010 (Panama City) and Miami-Dade County in February 2011; the latter outbreak is only $6 \mathrm{~km}$ from the state's commercial avocado production areas $(21,33)$.

Laurel wilt is a vascular disease that plugs the xylem, thereby impeding the flow of water and nutrients in affected trees (10). The first external, foliar symptoms of this disease on avocado are wilting of terminal leaves, which change from an oily green color to brown soon after wilting (20). Symptoms typically develop rapidly in affected portions of the tree. Unlike redbay, which retains dead leaves for a year or longer, avocado usually defoliates within 2 months of symptom onset. Internally, avocado sapwood is discolored reddish-brown to bluish-gray. Many of the symptoms of laurel wilt on avocado resemble those that are caused by other diseases or factors, such as freeze damage, Phytophthora root rot, Verticillium wilt, lightening, or overcropping.

This devastating disease has the potential to destroy avocado production in Florida (20). It has been estimated that financial loses could range from $\$ 27$ to 54 million in the absence of a reliable control strategy (22). The potential for rapid spread of this disease in avocado-growing regions is thought to be high, given the widespread distribution of native, susceptible host trees such as redbay, the natural movement of the vector $X$. glabratus, and human-mediated movement of pathogen- and vector-infested ma-

Corresponding author: R. Ehsani, E-mail: ehsani@ufl.edu

Accepted for publication 7 June 2012.

http://dx.doi.org/10.1094/PDIS-01-12-0030-RE

(C) 2012 The American Phytopathological Society terials. Laurel wilt is an emerging disease (8). There is a need for a comprehensive management approach that includes disease detection, fungicide application, vector control, sanitation (elimination of infected trees), and development of tolerant varieties (22).

As laurel wilt spreads into commercial avocado production areas, sanitation (i.e., removal of infected trees before new generations of infective $X$. glabratus emerge) will be an important tactic in laurel wilt management (20). Unfortunately, there is no available method to accurately and rapidly identify infected trees. The current method involves visual inspection of trees from a helicopter for identifying suspicious trees, followed by site visits to collect symptomatic wood. Reliable diagnosis by culture-based methods currently takes up to 2 weeks and is quite expensive to use on a large spatial scale. Clearly, a method for the rapid detection of laurel wilt would be most useful for combating this disease and making the detection process more cost effective. One such advanced, non-invasive sensing technique is visible-near infrared spectroscopy.

Visible-near infrared spectroscopy has been found to be a suitable technique for non-destructive disease detection in different crops $(12,16,17,26,34)$. Rumpf et al. (26) found that support vector machines with visible-near infrared spectra could classify diseased leaves (Cercospora leaf spot, sugar beet rust, and powdery mildew) with an accuracy of approximately $97 \%$. When the technique was used to classify individual diseases, an overall classification accuracy of $86 \%$ was achieved. Such technology offers a robust, costeffective, and user-friendly solution for plant disease detection. Similarly, Liu et al. (16) used hyperspectral reflectance data to evaluate the severity of rice brown spot (Bipolaris oryzae). The disease severity was grouped as S0 (healthy), S1 (1 to 25\%), S2 ( 25 to $50 \%)$, S3 (50 to $75 \%$ ), and S4 (>75\%) based on the percentage of rice brown spot symptoms per unit surface area of the leaf.

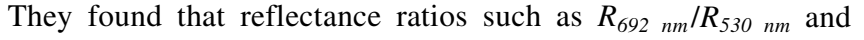
$R_{692 \mathrm{~nm}} / R_{732 \mathrm{~nm}}$ were highly correlated with disease severity and produced correlation coefficients of 0.9568 and 0.8690 , respectively. Jones et al. (12) assessed the bacterial spot disease severity in tomato leaves caused by Xanthomonas perforans using spectral signatures (200 to 2,300 nm) and found that absorbance from 750 to $760 \mathrm{~nm}$ contributed significantly to the prediction of disease severity. The visible-near infrared spectroscopy can also be ex- 
tended for aerial remote sensing of plant diseases. For example, studies on citrus greening detection in citrus using ground-based and aerial sensing have demonstrated the applicability of visiblenear infrared spectroscopy $(15,29)$.

In this study, we evaluated the use of visible-near infrared spectroscopy to detect laurel wilt. We compared the spectral patterns of leaves from symptomatic and asymptomatic avocado plants that were infected with $R$. lauricola, as well as those from plants that were not infected by the pathogen or were freeze-damaged. Linear discriminant analysis (LDA), quadratic discriminant analysis (QDA), Naïve-Bayes (NB) classifier, and bagged decision tree (BDT) were used for classification. This study provided useful information on optical sensing of laurel wilt under field conditions.

\section{Materials and Methods}

Host plants, inoculum preparation, and inoculation in greenhouse. Potted 'Simmonds', 'Choquette', and 'Bacon' avocado plants were obtained from a commercial nursery and maintained in an air-conditioned greenhouse (approximately $25^{\circ} \mathrm{C}$ ) at the University of Florida's Tropical Research and Education Center (TREC) in Homestead. Plants ( 1 to $1.5 \mathrm{~m}$ in height by $3 \mathrm{~cm}$ in diameter) consisted of clonal scions of cultivars grafted onto seedling rootstocks potted in 12-liter pots. Standard irrigation and fertilization practices were utilized (22).

A virulent isolate of $R$. lauricola (RL4) used in a previous study (22) was maintained on malt extract agar. Conidia were harvested from a 7-day-old culture by flooding with sterile, distilled water and gently scraping the surface with sterile glass rods. Conidial suspensions were adjusted to a final concentration of $10^{6}$ conidia $\mathrm{ml}^{-1}$. Approximately $15 \mathrm{~cm}$ above the graft union, a 2-mm-diameter wound was made by drilling at a $45^{\circ}$ downward angle, and 100 $\mu \mathrm{l}$ of the conidial suspension or sterile water (mock inoculated) was pipetted into the wound, which was then sealed with Parafilm.

Three plants were inoculated and one plant was mock inoculated (negative control) for each cultivar. Four leaves per cultivar were sampled from inoculated and mock inoculated plants at 3, 6, 12, 17 , and 45 days after inoculation (dai). Symptoms of laurel wilt developed by 12 dai and therefore, depending on the sample date, leaves from inoculated plants were categorized as follows: "asymptomatic" leaves, which were sampled at 3, 6, 12, 17, and 45 dai; or "symptomatic" leaves, which were sampled at 12, 17, and
45 dai. Leaves from mock inoculated plants never developed symptoms, and were referred to as "healthy", regardless of the sample date. Immediately after sampling, leaves were placed into Ziploc bags and shipped overnight on dry ice to the Citrus Research and Education Center, University of Florida, Lake Alfred. Within $48 \mathrm{~h}$ of sampling, the visible-near infrared reflectance spectra were collected from each leaf.

Field samples. In addition to leaves collected from the greenhouse as described above, field samples of healthy leaves (as negative controls) and freeze-damaged leaves (as possible false positives) from avocado trees were collected from a residential area in Lakeland, FL for which the disease was absent (i.e., plants were not infected).

Data collection. Spectral reflectance data were collected using a field-portable, handheld spectroradiometer (SVC HR-1024 Spectroradiometer; Spectra Vista Cooperation) with $4^{\circ}$ field of view in the spectral range 350 to $2,500 \mathrm{~nm}$, under controlled laboratory conditions (Fig. 1). In this study, a spectral range of 350 to 1,000 $\mathrm{nm}$ with $3.5-\mathrm{nm}$ spectral resolution was used, because detectors within this range would improve cost-effectiveness during sensor development. Two portable halogen work lamps $(500 \mathrm{~W})$ were used as a light source. The reference reflectance spectra were acquired using a white panel (Spectralon Reflectance Target, CSTMSRT-99-100; Spectra Vista Cooperation) in the presence of the light source. The detector response accounts for light conditions to compute the reflectance values from the leaves.

Table 1. Number of leaf spectra acquired during data collection ${ }^{\mathrm{a}}$

\begin{tabular}{lcccc}
\hline $\begin{array}{l}\text { Days after } \\
\text { inoculation }\end{array}$ & Healthy & $\begin{array}{c}\text { Asymptomatic- } \\
\text { infected }\end{array}$ & $\begin{array}{c}\text { Symptomatic- } \\
\text { infected }\end{array}$ & $\begin{array}{c}\text { Freeze- } \\
\text { damaged }\end{array}$ \\
\hline 3 & 17 & 18 & $\ldots$ & $\ldots$ \\
6 & 18 & 19 & $\ldots$ & $\ldots$ \\
12 & 18 & 25 & 6 & $\ldots$ \\
17 & 18 & 18 & 18 & $\ldots$ \\
45 & 56 & 38 & 40 & $\ldots$ \\
$\ldots$ & 87 & $\ldots$ & $\ldots$ & 81 \\
Total $^{\mathrm{b}}$ & 214 & 118 & 64 & 81 \\
\hline
\end{tabular}

${ }^{a}$ Spectra collected at 3,6,12,17, and 45 days after inoculation were from greenhouse avocado plants, while 87 healthy and 81 freeze-damaged spectra were collected from field trees.

${ }^{b}$ Total number of samples
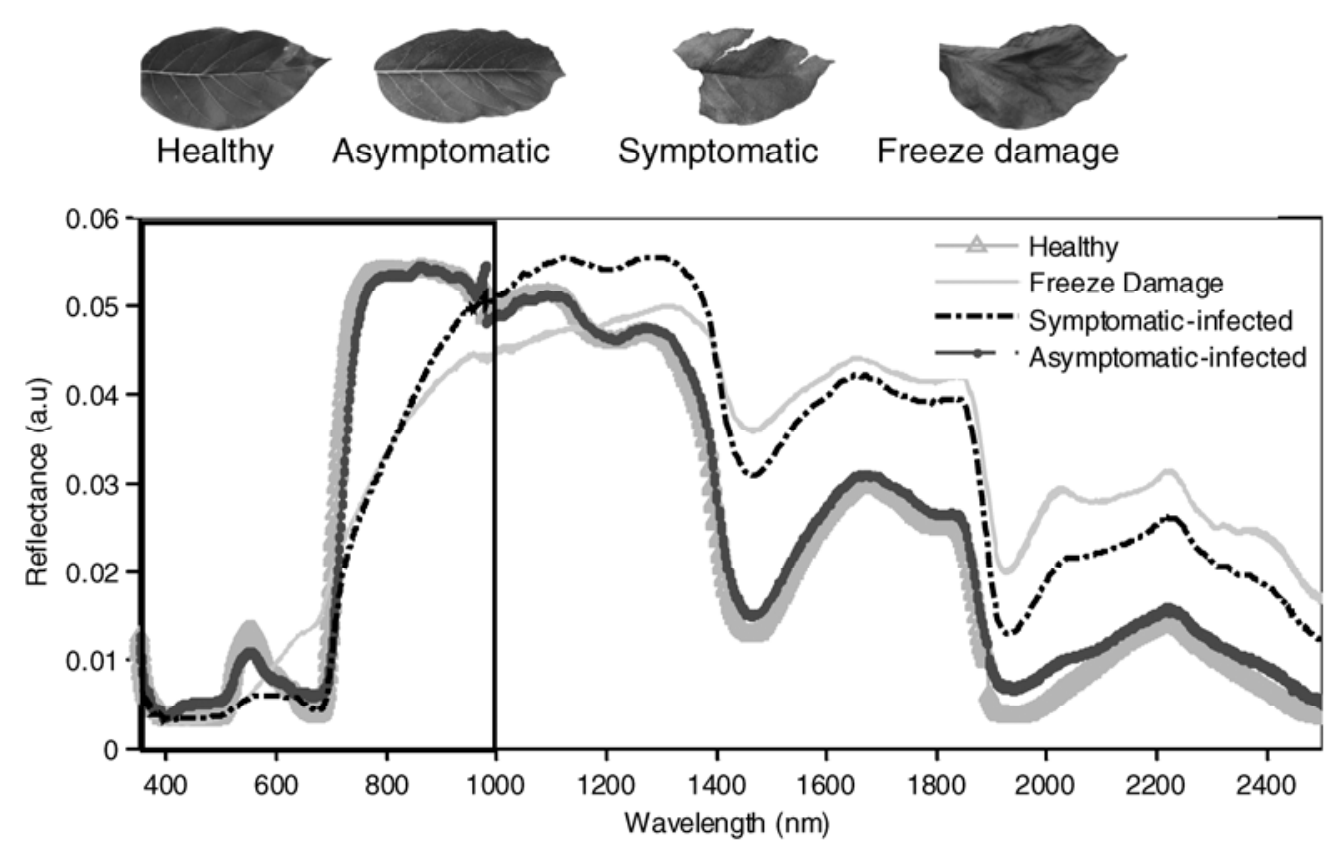

Fig. 1. Representative leaves from healthy, Raffaelea lauricola-infected (asymptomatic and symptomatic), and freeze-damaged avocado plants used in this study. Visiblenear infrared reflectance spectra representing each category of leaves are shown. 
Spectral data were collected from the adaxial surface of avocado leaves collected at 3,6,12,17, and 45 dai. The distance between the spectroradiometer and the leaves was approximately $0.5 \mathrm{~m}$. For each set of leaves, about six reflectance spectra were collected. In addition to data collected from the greenhouse study, reflectance spectra were also collected from field samples of healthy leaves or freeze-damaged leaves (Table 1).

Leaf spectra for healthy and asymptomatic-infected leaves were visually similar (Fig. 1). When symptomatic-infected and freezedamaged leaf spectra were compared with those of healthy leaves, a distinct spectral shift in the red-near infrared region was observed. Notably, a difference in the red-edge region distinguished symptomatic-infected from freeze-damaged leaves, wherein freezedamaged leaves exhibited a higher reflectance than symptomaticinfected leaves.

Data preprocessing. The Matlab 7.6 program (The MathWorks Inc.) was used for data preprocessing and data analysis. During preprocessing, spectral reflectance values were normalized using Euclidian norm function in Matlab. The normalized reflectance is computed by dividing individual reflectance values of each wavelength by the square root of sum of squares of all reflectance values of that spectrum (29). Following the normalization, the spectral values were binned at $10 \mathrm{~nm}$ wavelength intervals. The bandwidth of $10 \mathrm{~nm}$ was selected based on literature review and availability of commercial optical sensors (23). The total number of spectral reflectance features was 66 in the range of 350 to $1,000 \mathrm{~nm}$ with 10 nm band width.

Data analysis and classification. Statistics Toolbox 7 of Matlab was utilized in this study for classification. The principal component analysis (PCA) was used to reduce dimensionality and remove redundancy within the data. The PCA procedure involves the transformation of the data from a set of correlated to uncorrelated features called principal components (PCs). Using these PCs, PC scores are computed, with the first PC accounting for maximum possible variance within the data and PCs henceforth with remaining variance in a decreasing manner. The PC scores were used as input features into the classifiers. Classification studies were conducted to train different classifiers in order to classify the healthy and unhealthy leaves. During the classification, algorithms (classifiers) are developed based on a set of mathematical relationships using the input data (spectral features) of the training dataset in order to identify the category or class (healthy or unhealthy). Upon the algorithm development, the classifier uses the input features of test dataset and predicts its class or category. Finally, the performance of the developed classifier is tested with the actual class information of the test dataset.

The dataset was randomized and separated into two independent datasets, one for training and the other for testing, such that their ratio was 3:1 using the Matlab program. In other words, $75 \%$ of the spectral data features were used for developing the classifier and, then, the performance of the developed classifier was tested using $25 \%$ of the spectral data features (test dataset). The classifiers that were used for the analysis were LDA, QDA, NB, and BDT. Linear and non-linear classifiers were selected based on a literature review and preliminary evaluation. The LDA classifier develops a linear boundary by fitting a multivariate normal density with pooled covariance estimates for each class, whereas the QDA is a non-linear model that constructs non-linear boundary by fitting multivariate normal densities with covariance estimates separated by groups $(3,14,24,29)$. The LDA is a simple model that performs better while classifying small sample sizes and requires less computation time, whereas the QDA is more suited for a complex dataset. Similarly, the NB classifier is a simple model that estimates the probability or probability density for a given class using Bayes estimation to classify the unknown sample. In BDT, a classification tree is developed with bootstrap replicates to strengthen the developed tree used in classification. BDT has better stability than other classifiers, though the computation time is higher (27).

The classification analysis was performed in two ways. The two methods were chosen to answer the following questions. (i) Can asymptomatic and symptomatic $R$. lauricola-infected plants be identified? (ii) Can leaf symptoms caused by laurel wilt be differentiated from those of freeze damage? In the first method, the healthy and asymptomatic-infected spectra, and healthy and symptomatic-infected spectra of the three cultivars from the greenhouse, were analyzed as two different groups. The spectral data of three cultivars (Choquette, Simmonds, and Bacon) were combined for this study. During the classification, PC scores contributing to 99.9\% variability within the dataset were used as an input. A confusion matrix was derived from the classifiers, based on which factors (e.g., classification accuracy) indicating classifier performances were computed. Classification accuracy, specificity (healthy class classification accuracy), and sensitivity (diseased class classification accuracy) is defined as follows:

Accuracy $(\%)=[($ True Negative + True Positive $) /($ True Negative + False Negative + True Positive + False Positive $)] \times 100$

Specificity $(\%)=[$ True Negativel $($ True Negative + False Positive $)] \times 100$

Sensitivity $(\%)=[$ True Positive $/($ True Positive + False Negative $)] \times 100$

where "true positive" is defined as leaves from inoculated plants in the greenhouse (asymptomatic or symptomatic), correctly classified as diseased leaves; "true negative" is defined as healthy leaves from mock inoculated plants in the greenhouse, correctly classified as healthy leaves; "false positive" refers to healthy leaves wrongly classified as diseased leaves; and "false negative" refers to diseased leaves wrongly classified as healthy leaves. During the classification of healthy and asymptomatic-infected leaves, 249 samples were used for training and 83 samples were used for testing (total of 332 spectra) whereas, during the classification of healthy and symptomatic-infected leaves, 209 samples were used for training and 69 samples were used for testing (total of 278 spectra).

In the second method, the healthy (non-infected), asymptomaticinfected, and symptomatic-infected datasets from the greenhouse were combined with additional healthy and freeze-damaged field datasets to perform the classification. Thus, there were four classes: healthy (mock inoculated/non-infected), asymptomaticinfected (R. lauricola-inoculated plants, non-symptomatic leaves),
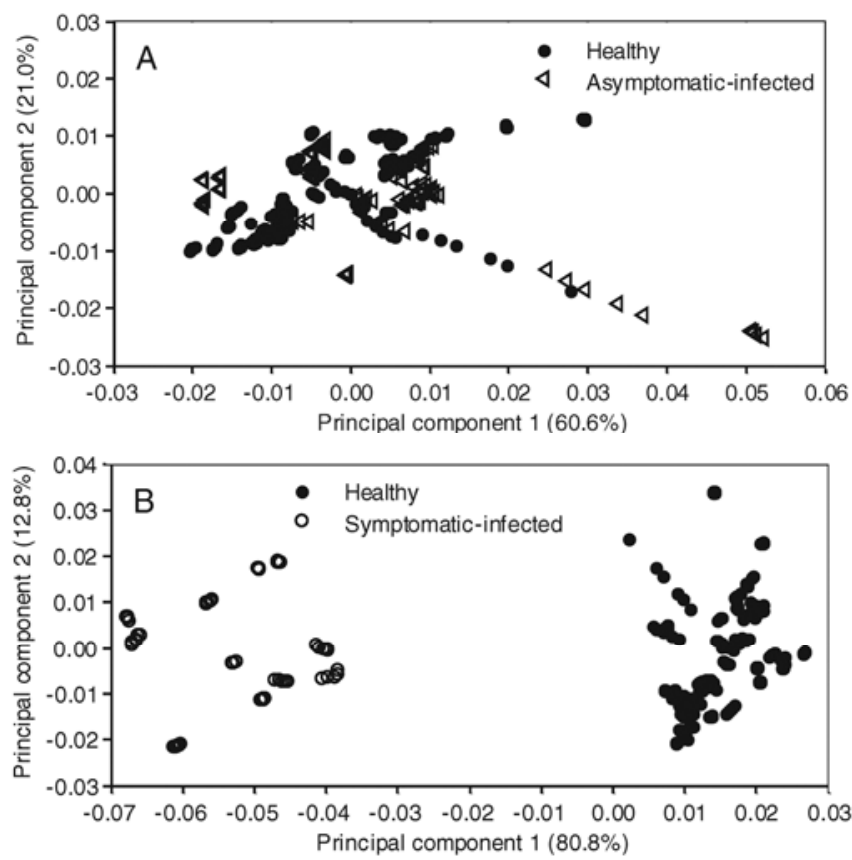

Fig. 2. Principal component analysis of $\mathbf{A}$, healthy versus asymptomatic-infected and $\mathbf{B}$, healthy versus symptomatic-infected spectra of avocado leaves. Each point represents individual spectrum. Percent variation explained by each principal component is shown in parentheses. 
symptomatic-infected ( $R$. lauricola-inoculated plants, symptomatic leaves), and freeze-damaged (healthy/non-infected) leaves. Similar to the first method, PC scores from visible-near infrared spectra (350 to $1,000 \mathrm{~nm}$ ) that contributed to $99.9 \%$ variance within the dataset were used as input features. The performance of the classifiers was assessed based on class recall (individual class accuracy) and precision. The class recall and precision are defined as follows:

Class Recall $(\%)=[$ True Positive $/($ True Positive + False Negative $)] \times 100$

Class Precision $(\%)=[$ True Positive $($ True Positive + False Positive $)] \times$ 100

where true positive refers to particular class/group (e.g., healthy) rightly classified, false negative refers to all data of that particular class wrongly classified (e.g., healthy classified as infected or freeze-damaged), and false positive refers to all other data wrongly classified as that particular class (e.g., R. lauricola-inoculated or freeze-damaged classified as healthy). The numbers of samples used for training and testing the classifiers were 358 and 119 , respectively (total of 477 spectra).

In addition to the entire spectra in the range 350 to $1,000 \mathrm{~nm}$ (second method), $100 \mathrm{~nm}$ spectral segments were analyzed to identify the spectral regions that have better potential to discriminate healthy, infected (asymptomatic and symptomatic), and freeze-damaged leaves. The spectral regions considered were $<400,400$ to 500,500 to 600,600 to 700,700 to 800,800 to 900 , and 900 to $1,000 \mathrm{~nm}$. Two PC scores that contributed to about $99 \%$ of the variance in most cases were used as input features in the classifiers.

The following vegetation indices were used that indicate greenness and light-use efficiency in the canopy $(5,6,11,25,30-32)$ : normalized difference vegetation index (NDVI), simple ratio index (SRI), red-edge (RE)-NDVI, modified red-edge (m)SRI, photochemical reflectance index (PRI), and red-green ratio index

Table 2. Performance of classifiers during classification of the visible-near infrared spectra of healthy and Raffaelea lauricola-infected asymptomatic leaves

\begin{tabular}{lccc}
\hline Classifiers $^{\mathbf{a}}$ & Accuracy (\%) & Specificity (\%) & Sensitivity (\%) \\
\hline LDA & 81 & 83 & 76 \\
QDA & 94 & 98 & 86 \\
NB & 90 & 100 & 72 \\
BDT & 95 & 100 & 86 \\
\hline
\end{tabular}

${ }^{\mathrm{a}}$ Linear discriminant analysis (LDA), quadratic discriminant analysis (QDA), Naïve-Bayes (NB) classifier, and bagged decision tree (BDT).
(RGRI). The vegetation indices were calculated using the following equations.

$$
\begin{aligned}
& N D V I=\frac{R_{795 \mathrm{~nm}}-R_{675 \mathrm{~nm}}}{R_{795 \mathrm{~nm}}+R_{675 \mathrm{~nm}}} \\
& S R I=\frac{R_{795 \mathrm{~nm}}}{R_{675 \mathrm{~nm}}} \\
& R E-N D V I=\frac{R_{755 \mathrm{~nm}}-R_{705 \mathrm{~nm}}}{R_{755 \mathrm{~nm}}+R_{705 \mathrm{~nm}}} \\
& m S R I=\frac{R_{755 \mathrm{~nm}}-R_{444 \mathrm{~nm}}}{R_{705 \mathrm{~nm}}-R_{444 \mathrm{~nm}}} \\
& P R I=\frac{R_{534 \mathrm{~nm}}-R_{565 \mathrm{~nm}}}{R_{534 \mathrm{~nm}}+R_{565 \mathrm{~nm}}} \\
& R G R I=\frac{\sum_{i=635 \mathrm{~nm}}^{655 \mathrm{~nm}}}{\sum_{i=495 \mathrm{~nm}}^{n} R}
\end{aligned}
$$

Analysis of variance (ANOVA) with Tukey's Studentized range test (multiple comparison tests) was performed using SAS (SAS 9.2; SAS Institute Inc.) to compare the average of vegetation indices between classes (477 samples). The $F$ values and probability were determined for each vegetation index. The results from ANOVA would indicate the vegetation indices that may be robust for diagnosing laurel wilt using aerial imaging. In addition to the PCA and classification of combined spectral data (all cultivars), the spectral data of individual cultivars were evaluated with PCA, separately. The numbers of spectra representing Choquette, Simmonds, and Bacon were 33, 22, and 21, respectively.

\section{Results}

Classification of leaves from healthy and $\boldsymbol{R}$. lauricola-inoculated plants from greenhouse. PCA showed that healthy and symptomatic-infected leaves were separated primarily by two PCs, which explained a combined total of $93.6 \%$ of the variation (Fig. 2). Samples in either category were clustered apart from each other on opposite ends of the x-axis. In contrast, healthy and asymptomatic-infected leaves were not clearly separated by PCA, where the two PCs contributed to $81.6 \%$ of the variation. During the

\begin{tabular}{|c|c|c|c|c|c|c|c|}
\hline Class & Healthy & $\begin{array}{c}\text { Asymptomatic- } \\
\text { infected }\end{array}$ & $\begin{array}{c}\text { Symptomatic- } \\
\text { infected }\end{array}$ & $\begin{array}{c}\text { Freeze- } \\
\text { damaged }\end{array}$ & True & $\begin{array}{c}\text { Class recall } \\
(\%)\end{array}$ & $\begin{array}{c}\text { Class precision } \\
(\%)\end{array}$ \\
\hline Healthy & 42 & 12 & 0 & 0 & 54 & 78 & 75 \\
\hline Asymptomatic-infected & 14 & 15 & 0 & 0 & 29 & 52 & 56 \\
\hline Symptomatic-infected & 0 & 0 & 16 & 0 & 16 & 100 & 89 \\
\hline Freeze-damaged & 0 & 0 & 2 & 18 & 20 & 90 & 100 \\
\hline Predicted & 56 & 27 & 18 & 18 & 119 & $76.5^{\mathrm{a}}$ & $\ldots$ \\
\hline
\end{tabular}
classification, scores of 13 and 10 PCs were used as input features for classifying healthy and asymptomatic-infected leaves, and healthy and symptomatic-infected leaves, respectively.

\begin{tabular}{|c|c|c|c|c|c|c|c|c|}
\hline \multirow[b]{2}{*}{ Class } & \multicolumn{4}{|c|}{ Class recall $(\%)$} & \multicolumn{4}{|c|}{ Class precision (\%) } \\
\hline & LDA & QDA & NB & BDT & LDA & QDA & NB & BDT \\
\hline Healthy & 78 & 96 & 91 & 100 & 75 & 88 & 78 & 98 \\
\hline Asymptomatic-infected & 52 & 76 & 52 & 97 & 56 & 92 & 75 & 100 \\
\hline Symptomatic-infected & 100 & 100 & 100 & 100 & 89 & 100 & 100 & 100 \\
\hline Freeze-damaged & 90 & 100 & 100 & 100 & 100 & 100 & 100 & 100 \\
\hline
\end{tabular}

Table 3. Confusion matrix and classification performance features generated using linear discriminant analysis during dataset testing while classifying four classes

a Overall classification accuracy.

Table 4. Classification results based on the spectral analysis ( 350 to $1,000 \mathrm{~nm}$ ) using different classifiers ${ }^{\mathrm{a}}$

${ }^{a}$ Linear discriminant analysis (LDA), quadratic discriminant analysis (QDA), Naïve-Bayes (NB) classifier, and bagged decision tree (BDT). 
When classification accuracies for spectra of different leaf classes were compared, symptomatic-infected leaves were distinguished from healthy leaves with $100 \%$ accuracy, specificity, and sensitivity, regardless of the classifiers. In contrast, the distinction of asymptomatic-infected and healthy leaves was less accurate, with the BDT resulting in the highest accuracy, 95\% (Table 2). In the latter comparisons, sensitivity was lower than specificity for all classifiers, indicating a large number of false negatives during classification. Among the classifiers, QDA, NB classifier, and BDT resulted in high classification accuracies. Both QDA and BDT resulted in maximum sensitivity of $86 \%$. Thus, in this study, the non-linear classifiers performed better than linear classifiers when classifying leaves from $R$. lauricola-infected plants.

Classification of healthy, infected, and freeze-damaged leaves from field and greenhouse. The classification performances were computed based on confusion matrix (Table 3). Scores of 10 PCs were used as the input into the classifiers. The class recall and precision rates were determined for four classes: healthy, asymptomatic-infected, symptomatic-infected, and freezedamaged leaves (Table 4). The overall classification accuracy of

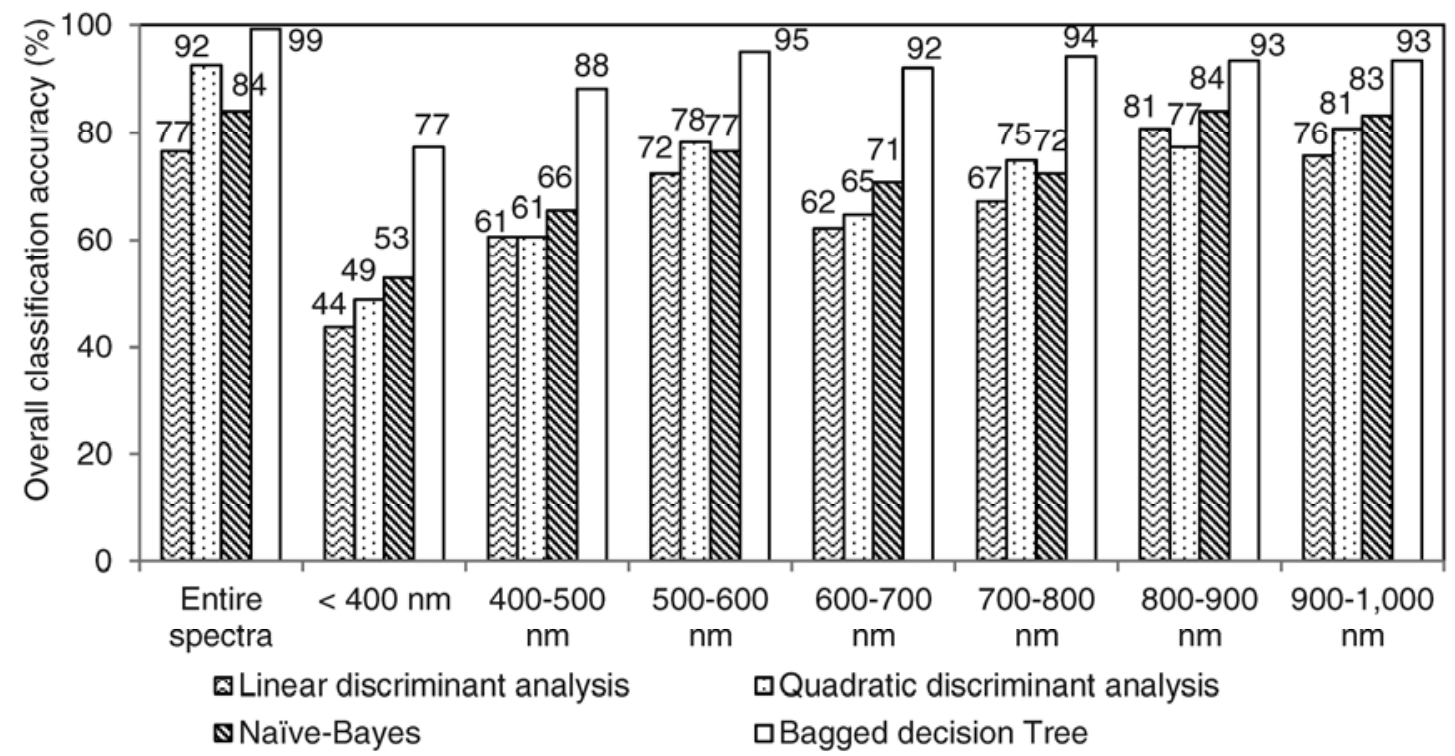

Fig. 3. Overall classification accuracy using the entire visible-near infrared spectra (350 to 1,000 nm) and specific spectral regions with $100 \mathrm{~nm}$ range with multiple classifiers.
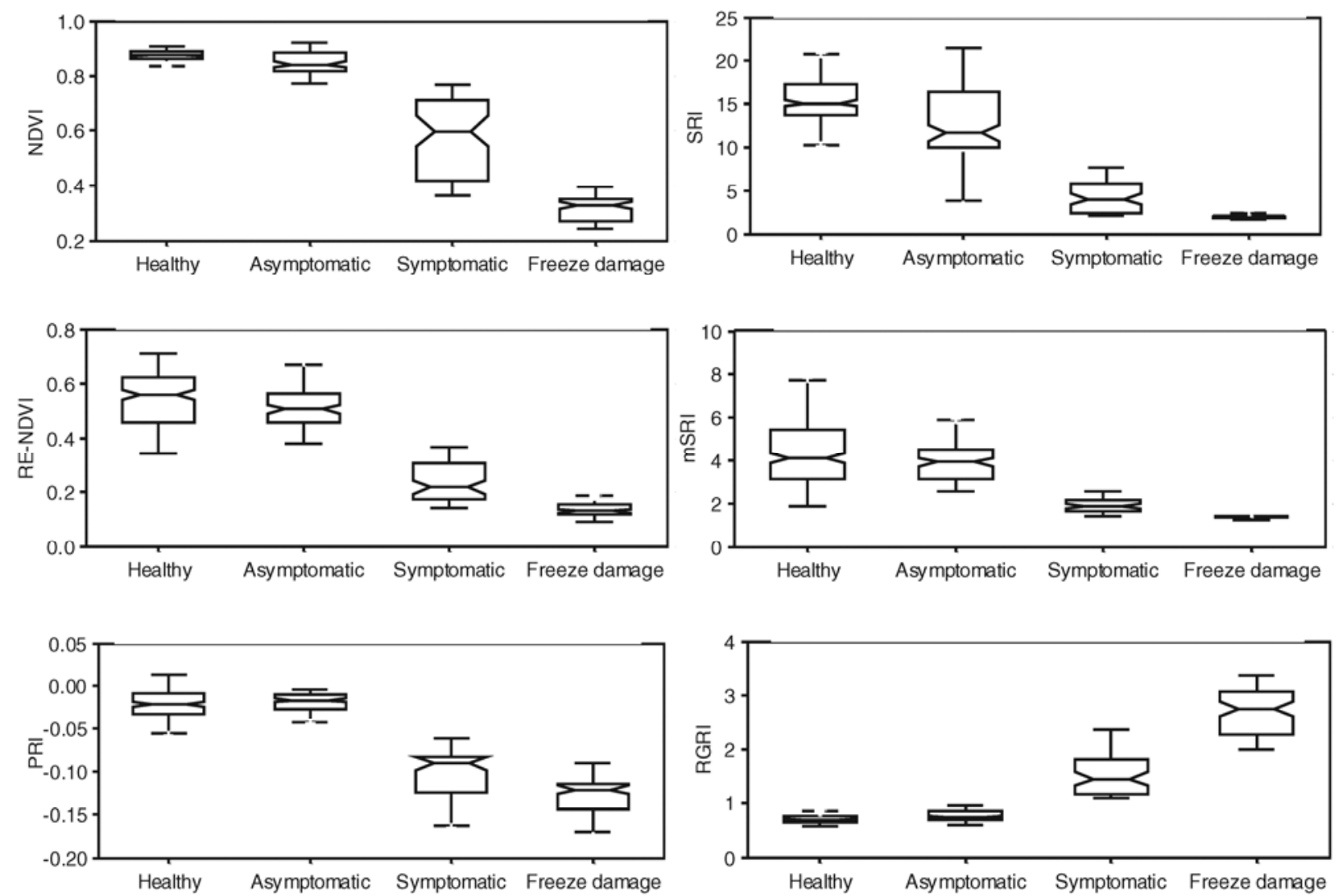

Fig. 4. Box plots showing variation in vegetation index computed using spectral features for healthy, laurel-wilt-infected (asymptomatic and symptomatic), and freezedamaged leaves. 
LDA, QDA, NB classifier, and BDT was 77, 92, 84, and 99\%, respectively.

Among the four classifiers, the BDT yielded the highest overall accuracy as well as recall and precision. The performance of QDA and BDT were better than NB classifier and LDA. With the exception of the LDA, all other classifiers correctly distinguished symptomatic-infected leaves from freeze-damaged leaves. When the class recall and precision values for individual classifier were compared, the lowest recall rate was for asymptomatic-infected leaves. The low recall rate of asymptomatic-infected leaves was due to the high number of false negatives (asymptomatic leaves classified as healthy), as observed previously. Therefore, though the classifiers were able to correctly distinguish $R$. lauricola infection from freeze damage, there was difficulty in classifying the asymptomatic-infected leaves; asymptomatic-infected leaves were often classified as healthy.

In addition to the entire spectra, the reflectance spectra were divided into regions of $100 \mathrm{~nm}$ in range to identify the spectral regions possessing high discriminability (Fig. 3). Two PC scores, computed using spectral features in a $100 \mathrm{~nm}$ range, contributing higher than $99 \%$ variance (except for spectral range of 500 to 600 $\mathrm{nm}$, which had $97 \%$ variance) were used as input features in the classifiers. BDT exhibited higher classification accuracies than the other classifiers in all the spectral regions. Although the classification accuracies were not as high as the entire spectrum (up to 1,000 $\mathrm{nm}$ ), the accuracies were higher than $88 \%$ in all the spectral re-
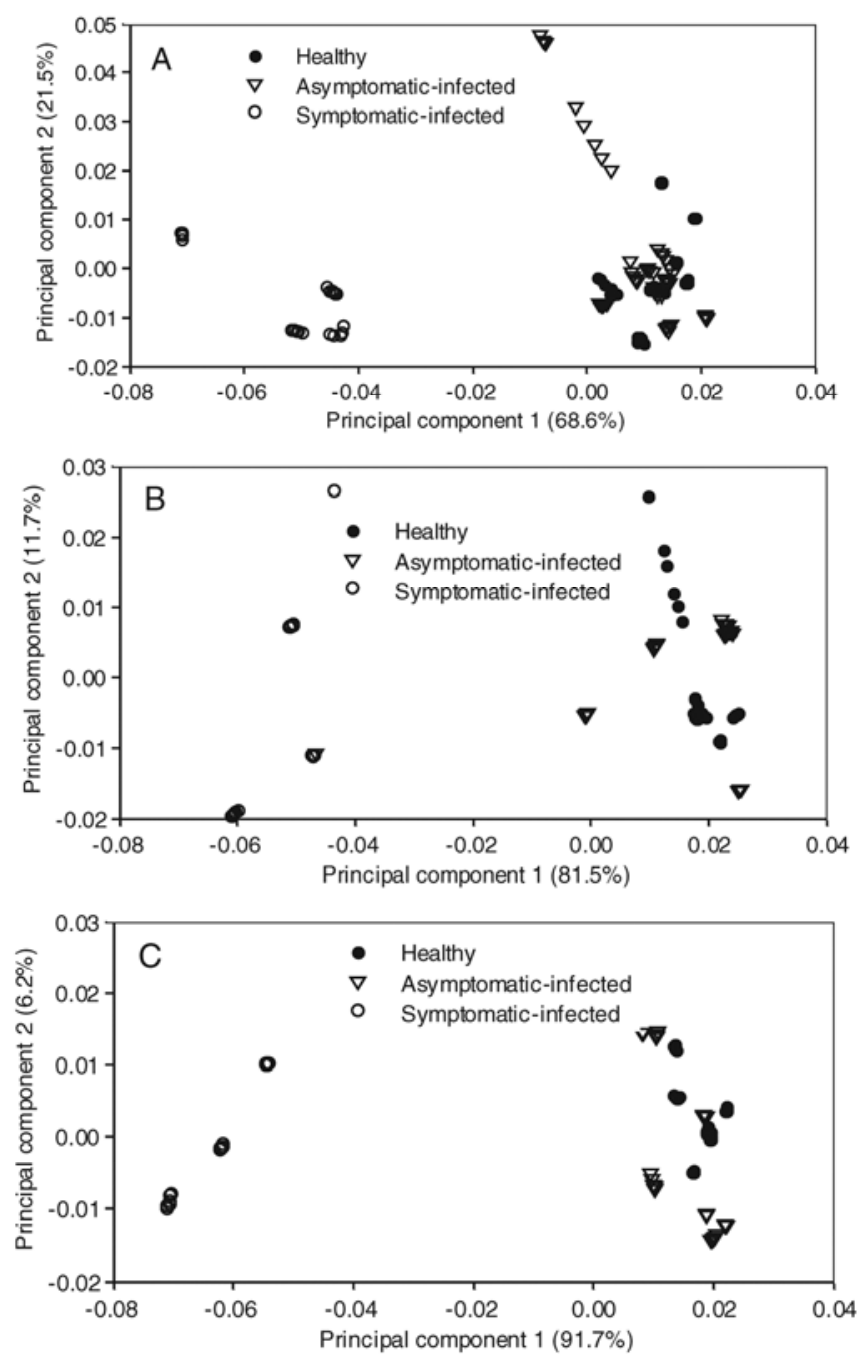

Fig. 5. Principal component analysis of healthy, asymptomatic-infected, and symptomatic-infected leaf spectra for A, 'Choquette'; B, 'Simmonds'; and C, 'Bacon' avocado. Each point represents individual spectrum. Percent variation explained by each principal component is shown in parentheses. gions except $<400 \mathrm{~nm}$. All four classifiers showed higher classification accuracies in the 500 to $600 \mathrm{~nm}$, red-edge, and near infrared regions of the visible-near infrared spectra than those of other visible regions.

Vegetation indices. Vegetation indices are tools that incorporate visible-near infrared spectral reflectance values that can be used as indicators of absorption of chlorophyll, which indicates "greenness" and stress conditions. With the exception of RGRI, vegetation indices were higher in healthy and asymptomatic-infected leaves than in symptomatic-infected and freeze-damaged leaves. The average NDVI, SRI, RE-NDVI, and mSRI were slightly higher in healthy leaves than in asymptomatic-infected leaves (Fig. 4). ANOVA indicated that the average vegetation index of at least one class was statistically different in all vegetation indices. The $F$ values of NDVI, SRI, RE-NDVI, mSRI, PRI, and RGRI were $1,024.08,373.03,529.86,165.78,714.82$, and 772.06, respectively. The $P$ values for all indices were $<0.0001$.

Further analysis using Tukey's multiple range comparison indicated that the average NDVI and SRI values were each different from one another for each leaf class $(\mathrm{df}=473)$. However, the average RE-NDVI, mSRI, PRI, and RGRI values were similar between healthy and asymptomatic-infected leaves, and between symptomatic-infected and freeze-damaged leaves.

PCA of reflectance spectra with respect to cultivar. PCA was performed individually for each cultivar to determine whether the distribution patterns of PC scores were different from those of the combined dataset (Fig. 5). For each cultivar, the first PC scores were lower in symptomatic-infected leaves than in asymptomaticinfected and healthy leaves, whereas asymptomatic-infected and healthy leaves were in a similar range. When the second PC scores were compared, all groups displayed similar variation. In total, these results were not significantly different from those shown in Figure 2, where the PCs were computed from the combined dataset. Although further data collection and analysis is needed, these results suggest that this approach for detecting laurel wilt may be generally used on avocado, regardless of the cultivar that is monitored.

\section{Discussion}

The current management strategy involves surveys of the commercial avocado production area in South Florida with helicopters to identify trees that may be affected by the disease, followed by ground-truthing, which is time-consuming as well as expensive. Therefore, it is essential to develop sensing technology to monitor the disease. In the present study, visible-near infrared spectroscopy was evaluated for detection of laurel wilt on avocado using statistical methods. The major findings of this research can be applied infield using similar visible-near infrared multiband spectroscopic techniques for aerial remote sensing and rapid identification of laurel wilt-affected trees. Others have used visible-near infrared spectroscopy to detect diseases in different crops $(9,28,29,35,36)$, although there has been no research on applicability of this technology to detect laurel wilt.

In this study, non-linear classifiers such as QDA and BDTs were able to classify some asymptomatic-infected leaves on infected plants, though the error was about $14 \%$. The visible-near infrared technique detected symptomatic-infected leaves much more accurately. A similar observation was found in several other studies $(2,7,19)$, including some that used visible-near infrared spectroscopy $(1,13)$; however, the classifier selection would depend on type of data and their application. It is possible that there is a non-linear relationship between spectral features and corresponding class (healthy or infected). Visible-near infrared spectroscopy has been used for detecting sugar beet diseases such as Cercospora leaf spot, leaf rust, and powdery mildew in asymptomatic-infected leaves with an accuracy of 65 to $90 \%$, depending on the disease stage and type of disease (26).

One of the challenges in the detection of laurel wilt symptomatic leaves is the similarity of symptoms to those caused by freeze damage; both conditions result in leaf necrosis. Thus, the visible-near 
infrared spectra of freeze-damaged leaves were also collected for classification. The results indicated that the sensing technique with classifier was able to differentiate symptomatic-infected from freeze-damaged leaves, though often the asymptomatic-infected leaves were classified as healthy. Given the uncertainty of symptom expression after infection, the classification of asymptomaticinfected and healthy leaves can be challenging. Further analysis of spectral bands indicated that the range of 500 to $600 \mathrm{~nm}$ (green, yellow) contributed to classification in addition to red-edge and near infrared regions that may represent physiological change in plants. Among the vegetation indices, it was found that the average NDVI and SRI values of each class were statistically different from each other. Both NDVI and SRI are sensitive to chlorophyll absorption and reflectance. Thus, the red and near infrared regions, including the red-edge region, were important for the classification of the groups into their respective category.

Although the present study demonstrated the potential for visible-near infrared detection of laurel wilt, further validation of this approach is needed. In particular, the spectra of avocado leaves that are killed or affected by laurel wilt need to be compared with those that are affected by other diseases and abiotic factors other than freeze damage. Ultimately, spectral reflectance data may enable the rapid and accurate identification of laurel wilt on avocado and enable the prompt removal of affected trees. Sanitation, wherein laurel-wilt-affected trees are destroyed prior to the production of infectious new generations of Xyleborus glabratus, may ultimately prove to be the most effective measure for managing this important new disease (22). Based on this study, a low-cost, robust, rugged multiband handheld sensor can also be developed for in-field detection of laurel wilt. In addition, the sensing technique evaluated in this work can further be extended for real-time aerial remote sensing using multispectral imaging to identify the diseased trees over a larger area in the field.

\section{Literature Cited}

1. Chen, L., Wang, J., Ye, Z., Zhao, J., Xue, X., Heyden, Y. V., and Sun, Q.2012. Classification of Chinese honeys according to their floral origin by near infrared spectroscopy. Food Chem. Online publication. doi:http://dx.doi.org/10.1016/j.foodchem.2012.02.156

2. Chen, Q., Cai, J., Wan, X., and Zhao, J. 2011. Application of linear/non-linear classification algorithms in discrimination of pork storage time using Fourier transform near infrared (FT-NIR) spectroscopy. LWT Food Sci. Technol. 44:2053-2058.

3. Dixon, S. J., and Brereton, R. G. 2009. Comparison of performance of five common classifiers represented as boundary methods: Euclidean distance to centroids, linear discriminant analysis, quadratic discriminant analysis, learning vector quantization and support vector machines, as dependent on data structure. Chemom. Intell. Lab. Syst. 95:1-17.

4. Fraedrich, S. W., Harrington, T. C., Rabaglia, R. J., Mayfield, A. E., Hanula, J. L., Eickwort, J. M., and Miller, D. R. 2008. A fungal symbiont of the redbay ambrosia beetle causes a lethal wilt in redbay and other Lauraceae in the Southeastern United States. Plant Dis.92:215-224.

5. Gamon, J. A., Serrano, L., and Surfus, J. S. 1997. The photochemical reflectance index: an optical indicator of photosynthetic radiation use efficiency across species, functional types and nutrient levels. Oecologia 112:492-501.

6. Gamon, J. A., and Surfus, J. S. 1999. Assessing leaf pigment content and activity with a reflectometer. New Phytol. 143:105-117.

7. Garrett, D., Peterson, D. S., Anderson, C. W., and Thaut, M. H. Comparison of linear, nonlinear, and feature selection methods for ECG signal classification. 2003. IEEE Trans. Neural Syst. Rehabil. Eng. 11:141-144.

8. Gramling, J. M. 2010. Potential effects of laurel wilt on the flora of North America. Southeast Nat. 9:827-836.

9. Huang, J., Liao, H., Zhu, Y., Sun, J., Sun, Q., and Liu, X. 2012. Hyperspectral detection of rice damaged by rice leaf folder (Cnaphalocrocis medinalis). Compt. Electron. Agric. 82:100-107.

10. Inch, S. A., and Ploetz, R. C. 2011. Impact of laurel wilt, caused by Raffaelea lauricola, on xylem function in avocado, Persea americana. For. Pathol. 42:239-245.

11. Jackson, T. L., Chen, D., Cosh, M., Li, F., Anderson, M., Walthall, C., Doriaswamy, P., and Hunt, E. R. 2004. Vegetation water content mapping using landsat data derived normalized difference water index for corn and soy- beans. Remote Sens. Environ. 92:475-482

12. Jones, C. D., Jones, J. B., and Lee, W. S. 2010. Diagnosis of bacterial spot of tomato using spectral signatures. Compt. Electron. Agric. 74:329-335.

13. Kim, J., Mowat, A., Poole, P., and Kasabov, N. 2000. Linear and non-linear pattern recognition models for classification of fruit from visible-near infrared spectra. Chemom. Intell. Lab. Syst. 51:201-216.

14. Krzanowski, W. J. 1988. Principles of Multivariate Analysis: A User's Perspective. Oxford University Press, New York.

15. Li, X., Lee, W. S., Li, M., Ehsani, R., Mishra, A., Yang, C., and Mangan, R. L. 2012. Spectral difference analysis and airborne imaging classification for citrus greening infected trees. Compt. Electron. Agric. 83:32-46.

16. Liu, Z. Y., Huang, J. F., and Tao, R. X. 2008. Characterizing and estimating fungal disease severity of rice brown spot with hyperspectral reflectance data. Rice Sci. 15:232-242.

17. Mahlein, A.-K., Steiner, U., Dehne, H.-W., and Oerke, E.-C. 2010. Spectral signatures of sugar beet leaves for the detection and differentiation of diseases. Prec. Agric. 11:413-431.

18. Mayfield, A. E., Peña, J. E., Crane, J. H., Smith, J. A., Branch, C. L., Ottoson, E. D., and Hughes, M. 2008. Ability of the redbay ambrosia beetle (Coleoptera: Curculionidae: Scolytinae) to bore into young avocado (Lauraceae) plants and transmit the laurel wilt pathogen (Raffaelea sp.). Fla. Entomol. 91:485-487.

19. Müller, K. R., Anderson, C. W., and Birch, G. E. 2003. Linear and nonlinear methods for brain-computer interfaces. IEEE Trans. Neural Syst. Rehabil. Eng. 11:165-169.

20. Ploetz, R. C., Harrington, T., Hulcr, J., Fraedrich, S., Smith, J. A., Inch, S. Kendra, P., Mayfield, A. E., Hanula, J., Rabaglia, R., Palmateer, A., Peña, J., Eskalen, A., Crane, J., Faber, B., Bostock, R., Harmon, C., Schnell, R., and Wingfield, W. 2011. Recovery plan for laurel wilt of avocado (caused by Raffaelea lauricola). National Plant Disease Recovery System. Homeland Security Presidential Directive Number 9 (HSPD-9). http://www.ars.usda. gov/research/docs.htm?docid $=14271$

21. Ploetz, R. C., Peña, J. E., Smith, J. A., Dreaden, T. J., Crane, J. H., Schubert, T., and Dixon, W. 2011. Laurel wilt is confirmed in Miami-Dade County, center of Florida's commercial avocado production. Plant Dis. 95:1589.

22. Ploetz, R. C., Pérez-Martínez, J. M., Smith, J. A., Hughes, M., Dreaden, T. J., and Fu, Y. 2012. Responses of avocado to laurel wilt, caused by Raffaelea lauricola. Plant Pathol. 61:801-808.

23. Ray, S. S., Jain, N., Miglani, A., Singh, J. P., Singh, A. K., Panigrahy, S. and Parihar, J. S. 2010. Defining optimum spectral narrow bands and bandwidths for agricultural applications. Curr. Sci. 98:1365-1369.

24. Roggo, Y., Chalus, P., Maurer, L., Lema-Martinez, C., Edmond, A., and Jent, N. 2007. A review of near infrared spectroscopy and chemometrics in pharmaceutical technologies. J. Pharmaceut. Biomed. 44:683-700.

25. Rouse, J. W., Haas, R. H., Schell, J. A., and Deering, D. W. 1973. Monitoring vegetation systems in the Great Plains with ERTS. Pages 309-317 in: Third ERTS Symp. NASA SP-351 I.

26. Rumpf, T., Mahlein, A.-K., Steiner, U., Oerke, E.-C., Dehne, H.-W., and Plümer, L. 2010. Early detection and classification of plant diseases with support vector machines based on hyperspectral reflectance. Compt. Electron. Agric. 74:91-99.

27. Sankaran, S., and Ehsani, R. 2012. Detection of Huanglongbing disease in citrus using fluorescence spectroscopy. Trans. ASABE 55:313-320.

28. Sankaran, S., Mishra, A., Ehsani, R., and Davis, C. 2010. A review of advanced techniques for detecting plant diseases. Compt. Electron. Agric. $72: 1-13$.

29. Sankaran, S., Mishra, A., Maja, J. M., and Ehsani, R. 2011. Visible-near infrared spectroscopy for detection of Huanglongbing in citrus orchards. Compt. Electron. Agric. 77:127-134.

30. Sellers, P. J. 1985. Canopy reflectance, photosynthesis and transpiration. International J. Remote Sens. 6:1335-1372.

31. Sims, D. A., and Gamon, J. A. 2002. Relationships between leaf pigment content and spectral reflectance across a wide range of species, leaf structures and developmental stages. Remote Sens. Environ. 81:337-354.

32. Tucker, C. J. 1979. Red and photographic infrared linear combinations for monitoring vegetation. Remote Sens. Environ. 8:127-150.

33. U.S. Dep. Agric. 2010. Distribution map for laurel wilt. http://www.fs. fed.us/r8/foresthealth/laurelwilt/dist_map.shtml

34. Wu, D., Feng, L., Zhang, C., and He, Y. 2008. Early detection of Botrytis cinerea on eggplant leaves based on visible and near-infrared spectroscopy. Trans. ASABE 51:1133-1139.

35. Xu, H. R., Ying, Y. B., Fu, X. P., and Zhu, S. P. 2007. Near-infrared spectroscopy in detecting leaf miner damage on tomato leaf. Biosyst. Eng. 96:447-454.

36. Zhang, M., Qin, Z., Liu, X., and Ustin, S. L. 2003. Detection of stress in tomatoes induced by late blight disease in California, USA, using hyperspectral remote sensing. Int. J Appl. Earth Obs. Geoinf. 4:295-310. 